\title{
ESTRATÉGIAS DE REDUÇÃO DA VARIABILIDADE DE PROCESSOS NA INDÚSTRIA CALÇADISTA
}

\section{STRATEGIES FOR REDUCING THE PROCESSES VARIABILITY IN THE FOOTWEAR INDUSTRY}

\author{
Taise Gross ${ }^{1}$, Diego Augusto de Jesus Pacheco2, Fernando Elemar Vicente dos \\ Anjos $^{3}$, Carlos Fernando Jung ${ }^{4}$
}

\begin{abstract}
This article presents a case study of a Brazilian company, operating in the footwear sector, located in Parobé city, in the Valley of Paranhana, in the state of Rio Grande do Sul. The process analyzed showed high failure rates of manufactured batches and by quality analysis tools, the Statistical Process Control tools were applied to analyze the variability. The tools applied were the control charts, process capacity and capability analysis, histogram, Pareto chart and cause and effect analysis. The main results of the study were the reduction of the rejection indices of the process to zero and improvement in the capacity index from 0,424 to 1,52 , demonstrating the reduction of the sources of variation of the production process and reaching the objectives The research. This research can be used by academics and professionals to improve the competitive performance of footwear companies.
\end{abstract}

Keywords: Statistical Process Control, Synthetics laminates, Process capability, Analysis of cause and effect.

\section{RESUMO}

Este artigo apresenta um estudo de caso de uma empresa brasileira, atuante no setor calçadista, localizada na cidade de Parobé, no Vale do Paranhana, no estado do Rio Grande do Sul. O processo analisado demonstrava altos índices de reprovação dos lotes fabricados e através da análise de ferramentas da qualidade, aplicaram-se as ferramentas do controle estatístico de processo para analisar a variabilidade. As ferramentas aplicadas foram as

\footnotetext{
${ }^{1}$ Bacharel em Gestão da Qualidade, Faculdades Integradas de Taquara, orcid.org/0000-0002-7980-2262

${ }^{2}$ Coordenador e professor do curso de Engenharia de Produção na UniRitter, orcid.org/0000-0003-4453-7216

${ }^{3}$ Professor do curso de Engenharia de Produção da Faculdade Anhanguera de Caxias do Sul, orcid.org/0000-00029096-2806

${ }^{4}$ Coordenador e professor do curso de Engenharia de Produção, Faculdades Integradas de Taquara, orcid.org/00000002-6317-8338
}

- Autor para correspondência: diego_pacheco@uniritter.edu.br 
cartas de controle, análise de capacidade e capabilidade do processo, histograma, gráfico de Pareto e a análise de causa e efeito. Como principais resultados do estudo, identificaram-se a redução dos índices de rejeição do processo para zero, e melhoria no índice do capabilidade de 0,424 para 1,52 , demonstrando a redução das fontes de variação do processo produtivo e atingindo-se os objetivos da pesquisa. Essa pesquisa pode ser usada por acadêmicos e profissionais para melhorar o desempenho competitivo de empresas calçadistas.

Palavras-chaves: Controle estatístico do processo; Laminados sintéticos; Capabilidade de processo; Análise de causa e efeito.

\section{INTRODUÇÃO}

As empresas têm investido cada dia mais em tecnologias e mão de obra especializada, visando melhorar seus produtos e processos para obter padronização, estabilidade, e incremento na garantia de qualidade e satisfação do cliente, fortalecendo fatores competitivos para a organização.

Aindústria calçadista inclui-se nesta necessidade de sempre buscar melhorias e oportunidades para o seu processo. Nesta indústria, um dos principais materiais aplicados é o laminado sintético, produto foco deste estudo, que ingressou no mercado calçadista brasileiro por volta de 1990, para aplicações em forro e reforço de diversos tipos de calçados. Nos anos de 2000, o laminado sintético passou a ser o material mais utilizado nos cabedais, chegando a esse status devido a sua versatilidade de aplicação, aproveitamento para maior estrutura, padronização e uniformidade (ASSINTECAL, 2016).

Atualmente no Brasil, existe um grande mercado de laminados sintéticos, com grande representatividade mundial neste segmento. Isso ocorre devido à grande indústria calçadista no Brasil, tornando-o um dos maiores consumidores mundiais deste insumo. Segundo dados da ASSISTENCAL (2016), no Brasil existem diversos diferenciais competitivos, mantendo-se forte em relação a outros concorrentes mundiais, mas ainda sendo fortemente pressionado pela China, pela sua capacidade de produzir a custos bem mais baixos.

Existe na fabricação de laminado sintético a necessidade de controle rigoroso de matériaprima, pois ele garante que o produto fabricado atenda às especificações do cliente, com qualidade e custo adequado. Com isso, o laminado terá competitividade no mercado (ABICALÇADOS, 2016). Gejdos (2015) afirma que o controle estatístico da qualidade é uma ferramenta de melhoria continua e que as cartas de controle de processos, os índices de capabilidade e o histograma demonstram as potenciais variabilidades do processo e em quais características devem ser dispostos esforços para a melhoria do processo e a satisfação do cliente.

A pesquisa de Bracke e Backes (2015) cita que a estabilidade do processo pode ser mensurada através das cartas de controle e a capacidade do processo mensuram a condição que o processo tem em cumprir as especificações determinadas para o processo, sendo que existe uma relação direta entre a rejeição nos processos e este índice. Para melhorar a estabilidade do processo, se faz necessário propor ações e aplicações de combinações de ferramentas para detectar as causas das falhas e estabilizar o processo. Nesse contexto, o tema da estabilidade de processos na fabricação de laminados sintéticos será discutido no 
presente estudo.

A empresa que é o objeto deste estudo de caso apresentava historicamente problemas de alto índice de rejeição nos processos produtivos de laminados sintéticos por variação da cor do laminado, gerando altos índices de sucata, altos custos de fabricação, atrasos de entregas aos clientes, problemas de imagem da empresa no mercado e perda de competitividade. Entendeu-se que essa empresa precisava buscar soluções a partir da eliminação desses problemas para elevar sua competitividade. Como consequência, foi constatado a partir da literatura que a aplicação das ferramentas do Controle Estatístico de Processos (CEP) poderiam reduzir a variabilidade do processo. Assim, o problema de pesquisa é o seguinte: como reduzir a variabilidade de processos do processo produtivo de laminados sintéticos melhorando os índices de pós-vendas?

Nesse sentido, o principal objetivo desse estudo foi analisar como a aplicação das ferramentas do CEP apoiaram as pessoas envolvidas a tratar o problema na organização, análises de problemas e obtendo como resultado a eliminação da fonte de variação do processo que impactam nos índices de rejeição, nos clientes e no pós-vendas.

\section{REFERENCIAL TEÓRICO}

No referencial teórico deste artigo será abordada a importância para o cliente sobre a padronização visual dos produtos, o processo de fabricação de laminados para a indústria calçadista e o controle estatístico do processo.

Para Farias (2012), a satisfação do cliente é o principal resultado esperado pela organização, sendo ela a razão da permanência da empresa no mercado, gerando a possibilidade de perpetuação do negócio. A competitividade é realidade em todas as indústrias, cada vez maior no setor calçadista, que por sua vez, busca fortalecer fatores de qualidade das matériasprimas, processos e dos produtos. A competição gera maior necessidade por conformidade nos processos e padronização em todas as etapas, desenvolvimento de novos materiais e busca de todos os tipos de alternativas de ferramentas e métodos de trabalho.

Um dos fatores com alta importância na indústria calçadista é a matéria prima que ela utiliza sendo que uma das alternativas é a aplicação de laminados sintéticos nos seus produtos. Ele tem custo mais baixo e permite a fabricação de calçados substituindo o couro como matériaprima principal. Nesta indústria, as cores desempenham funções importantes, principalmente pela percepção dos clientes, as cores são capazes de influenciar a decisão de compra do cliente ASSITENCAL (2016).

Conforme a ASSITENCAL (2016), os laminados sintéticos são materiais formados por uma base e uma ou algumas camadas de cobertura, deixando-o com propriedades físico-mecânicas para a aplicação. Alguns tipos de laminados podem ter acabamentos complementares e mudanças no seu aspecto superficial.

O seu processo de fabricação é denominado espalmagem. A espalmagem consiste na deposição de camadas de pastas confeccionadas a partir de polímeros de PU, poliuretano, PVC, plastisol, diretamente sobre um papel siliconado e acoplamento de um substrato, que pode ser telas, malhas, não tecidos ou bases coaguladas de PU. A Figura 1 demonstra um 
sistema de espalmagem em três camadas, na qual se observa a presença de três sistemas de aplicação de pasta e três fornos de gelificação, fusão e cura. (ASSITENCAL, 2016).

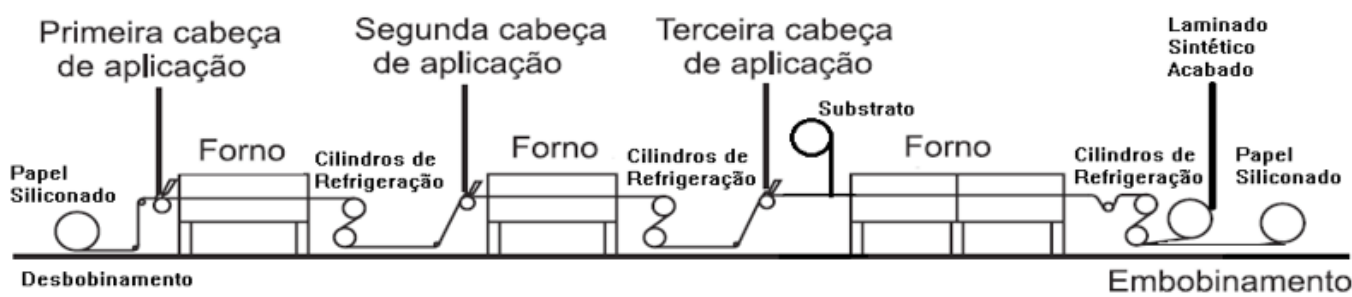

Figura 1. Demonstração do fluxo do processo de espalmagem.

Fonte: dados da pesquisa.

Um processo típico e simplificado de espalmagem direta é composto por:

- Seções de aplicação de pasta, consistindo em um dispositivo de deposição com sistema de facas, também conhecido como cabeça de aplicação.

- Fornos de cura, gelificação e fusão da pasta.

- Unidades de resfriamento.

- Sistemas de acoplamento do substrato.

- Dispositivos para desembobinar e tensionar o substrato e o papel.

- Sistema de embobinamento do espalmado.

Para os laminados de Poliuretano (PU), o material de base impregna a camada têxtil. Sobre esta é aplicada uma camada de cobertura que tem a função de melhorar o aspecto superficial dos materiais. Existem materiais com menor grau de impregnação. Para os laminados de PVC, a camada têxtil é novamente apenas adesivada ao restante do corpo de PVC, podendo ser mais ou menos impregnada. A aplicação de acabamentos complementares, como lacas, estampas diversas e transfer, melhora ainda mais o aspecto superficial. Porém, cuidados especiais são necessários para não danificar esses acabamentos (ASSINTECAL, 2016).

O arranjo mais comum nesse caso consiste na lâmina posicionada em linha, sendo a pasta do polímero adicionada a um reservatório sobre o papel siliconado que, em movimento, promove a formação de uma massa diante da lâmina, sendo essa espalhada ao longo do papel siliconado. A distância entre a lâmina e o papel determina a espessura e a gramatura do polímero espalmado. A temperatura dos fornos necessita ser controlada por zonas, considerando a intensidade e a distribuição de calor para promover a cura uniforme de cada camada do polímero aplicado.

Depois de cada camada de polímero ser depositada nas cabeças de aplicações sobre o papel, a mesma sofre o processo de cura e fusão dentro do forno, formando uma camada resistente. Após aplicação da última camada, antes de entrar no forno, é acoplado um substrato. O conjunto todo entra na última estufa onde ocorre a cura final. Por fim, o laminado sintético é desmoldado do papel siliconado, copiando a textura do mesmo.

Em concordância com Montgomery (2009), independente do que está sendo produzido, todo processo está sujeito à ação da variabilidade natural. Um dos primeiros estudos sobre qualidade com foco em processos foi conduzido pelo norte-americano Walter Shewart. Ele analisou diversos processos e percebeu que todos tinham variações e as classificou como casuais (comuns ou naturais) e atribuídas (especiais ou assinaláveis). 
Ainda de acordo com Montgomery (2009), o objetivo da aplicação de métodos de CEP é monitorar a variabilidade do processo em tempo real e agir em casos em que ocorra a desestabilização do processo ou o mesmo apresente variabilidade. A aplicação das ferramentas do CEP de maneira adequada tende a obtenção de um processo mais estável, estabelecendo o processo em níveis de controle.

Normalmente se entende que o CEP é um conjunto de sete ferramentas para resolver problemas, que pode ser aplicado a qualquer tipo de processo. Mas seu principal objetivo é buscar a estabilidade de um processo e melhorar a capacidade por meio da redução da variabilidade. As sete ferramentas aplicadas no CEP são, segundo Montgomery et al. (2004):

1. Histograma

2. Gráfico de Pareto

3. Diagrama de causa e efeito

4. Diagrama de concentração de defeito

5. Diagrama de dispersão

6. Folha de verificação

7. Gráficos de controle.

Alencar et al. (2005) destaca que o CEP é uma ferramenta de monitoramento online da qualidade que permite uma descrição precisa do comportamento de um processo. Ela identifica a variabilidade e possibilita seu controle ao longo do tempo, por meio de uma coleta contínua de dados e da análise e bloqueio mediante ações corretivas de possíveis causas especiais.

Todas as ferramentas do CEP são importantes, mas as cartas ou gráficos de controle são as principais ferramentas utilizadas no CEP. Elas também são denominadas cartas de Shewhart, sendo possível detectar os desvios nos parâmetros definidos para o processo, que causam a não conformidade, levando, dessa forma, a uma diminuição, na fabricação, de produtos fora de especificações e a uma redução dos custos de produção (Alencar et al., 2005)

Muler et al. (2015) cita que os gráficos de controle de processos são uma importante ferramenta para monitorar as variáveis de controle de processos, apoiando na condição de separar causas comuns das causas especiais. De acordo com Mancuso e Werner (2014), os gráficos de controle são ferramentas passíveis de percepção de problemas no processo, gerando a oportunidade de perceber anomalias no processo.

Mancuso e Werner (2014) também afirmam que as aplicações das ferramentas do CEP de maneira estrutura apoiam as organizações na percepção dos problemas e localizar as suas causas fundamentais, facilitando a definição das ações para a eliminação das fontes de variabilidade do processo.

\section{MÉTODO}

Esta pesquisa é relevante para o segmento das indústrias calçadistas, pois ao pesquisar no período de 1995 a 2016 por referências nas principais bases científicas (Scopus, Web of Science, DOAJ, REDIB, LATINDEX, SCIELO, e REDALYC) pela combinação dos termos de busca "laminados", "laminados sintéticos", "calçado", "indústria calçadista" com os termos "CEP" e "Controle Estatístico de Processos" nos campos de busca Título e Resumo não foi encontrado nenhum estudo relacionado diretamente ao objetivo da pesquisa. Para abordar 
o assunto específico do problema de rejeição de qualidade na inspeção de cor de laminados sintéticos e as implicações sobre o retorno de produtos de pós-vendas, o delineamento metodológico considerou fontes primárias de coletas de dados.

Para a execução deste estudo, aplicou-se o método de pesquisa estudo de caso. Conforme pondera Miguel et al. (2010), o estudo de caso tem um caráter empírico que busca interpretar a realidade por meio de uma análise profunda de um ou mais objetos de estudo. O objeto de estudo o caso de uma empresa que fabrica laminados sintéticos e que tem um alto índice de reprovação de seus materiais fabricados. A pesquisa tem abordagem qualitativa e natureza aplicada. Para realização dos estudos foram seguidos os passos:

- Utilização do diagrama de Pareto para analisar os índices de rejeição por cor fabricada em um período de três meses.

- Delimitação do estudo para aplicação das ferramentas do CEP.

- Elaboração do histograma dos dados coletados da amostra.

- Plotar os dados em gráficos de controle de processos de médias.

- Análise do CP e CPk do processo.

- Realizar a análise de causa e efeito.

Para a coleta de dados, analisou-se o índice de rejeição dos últimos 20 lotes de fabricação das cores de maior demanda (branco, cinza, preto, castanho, vermelho, amarelo, azul e verde). A cor que obtiver o maior índice de rejeição será a delimitação do estudo. O equipamento utilizado para a coleta de dados é o espectrofotômetro. Ele faz a leitura e compara com um padrão de cores que consta do seu banco de dados. Na Figura 2, segue a demonstração do equipamento.

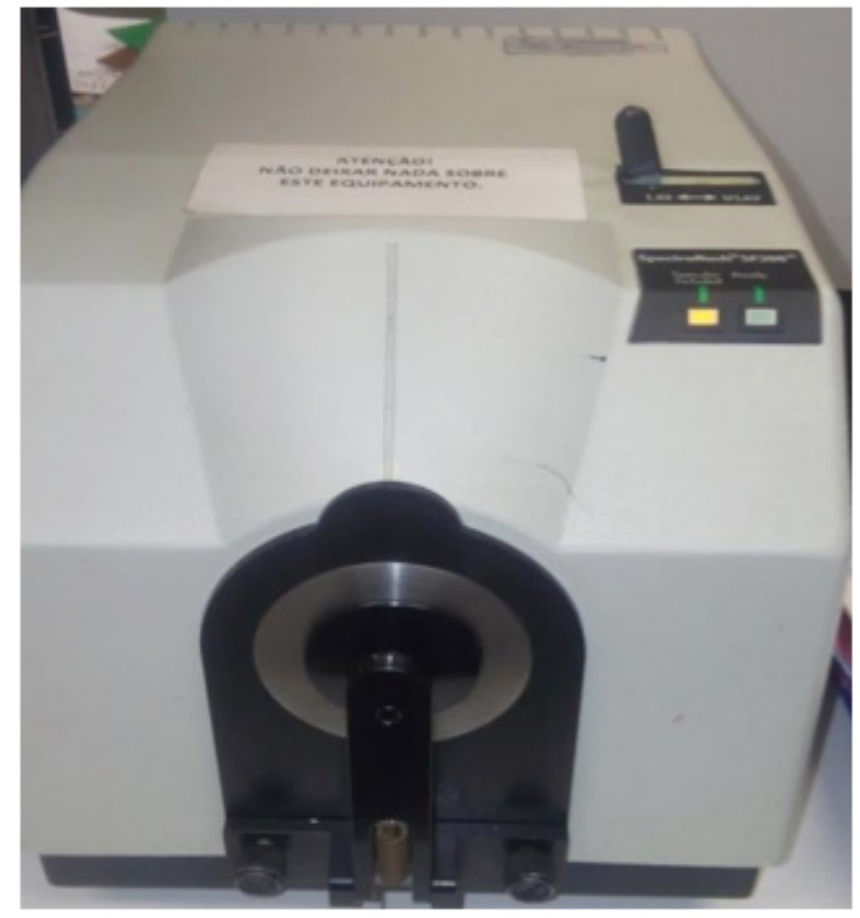

Figura 2. Espectrofotômetro.

Fonte: dados da pesquisa. 
Para a condição de aprovação ou reprovação da cor fabricada, utiliza-se o conceito de afastamento para determinar se a cor está conforme ou não. Baseando-se na cor padrão registrada no equipamento, ou seja, a cor definida como perfeita, ela é registrada como valor zero no equipamento. O equipamento funciona com a classificação descrita a seguir:

- 0,00 a 0,50 - classificam-se com bom, sinalizado pela cor verde;

- 0,51 a 0,99 - classificam-se como aceitável, sinalizado pela cor azul I;

- 1,00 parta cima - fora de cor, não aceita, sinalizado pela cor vermelha;

Dessa maneira, quando um resultado é mais próximo a zero, significa que a cor está se aproximando da cor definida como padrão (ideal). O inverso, ou seja, quando valor medido se afastar de zero, estará se distanciando do padrão estabelecido.

Após a definição da cor com maior índice de rejeição, utilizar-se-á uma amostra de tamanho 30 (próximos 30 lotes de produção), para monitorar o comportamento estatístico do processo. O tamanho da amostra foi definido baseando-se no histórico de fabricação, onde a empresa em média fabrica 30 lotes a cada 90 dias de laminado sintético.

\section{RESULTADOS}

Primeiramente, coletaram-se os dados históricos dos últimos lotes de fabricação, das oito cores que a empresa produz, para buscar entender onde se apresentava o maior índice de reprovação do material. Quando compilados os índices de reprovação das cores fabricadas, obtiveram-se os dados apresentados Figura 3.

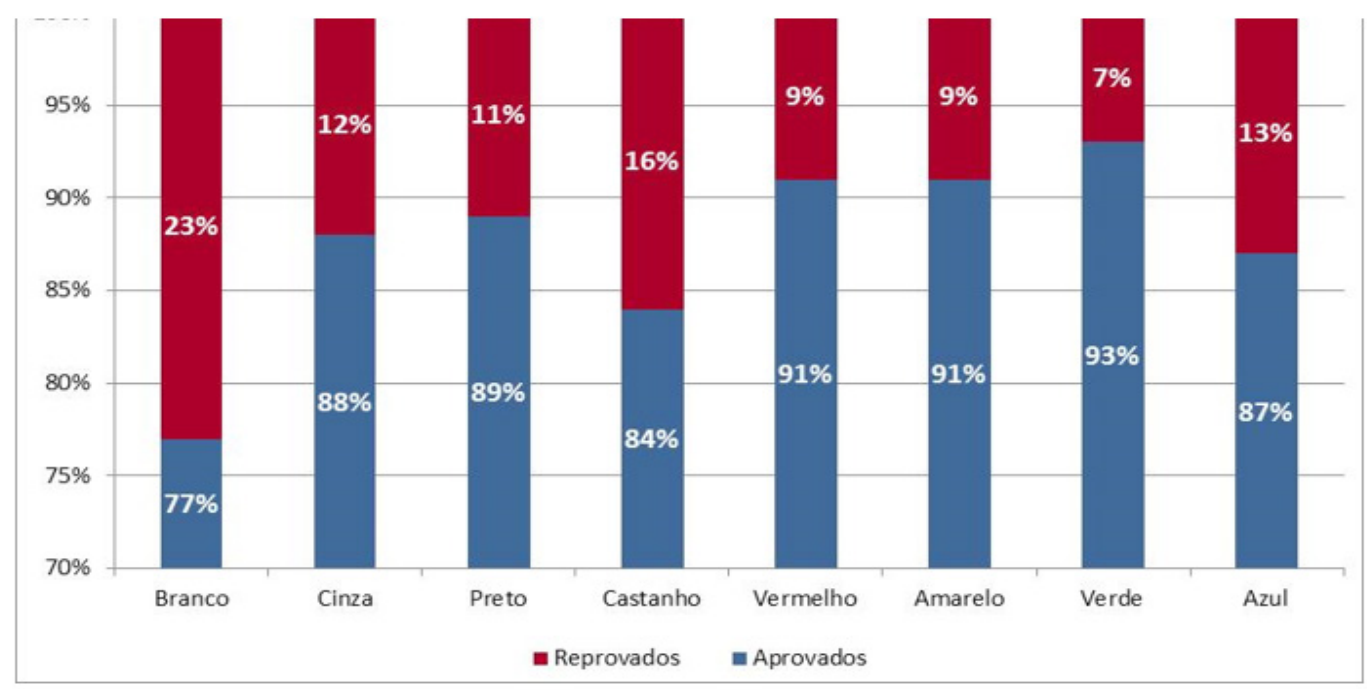

Figura 3. Índice de reprovação por cor no processo de fabricação de laminados sintéticos.

Analisando os índices de reprovação por cores fabricadas de laminados sintéticos, percebeuse que a maior incidência de rejeição está no laminado sintético branco, tendo $23 \%$ de 
rejeição nos lotes fabricados. Como definido na delimitação do estudo, aplicou-se o estudo de CEP e suas ferramentas nessa cor para identificar quais são as causas que estão gerando a rejeição na fabricação desse laminado.

Após a definição da delimitação do estudo, monitoraram-se os próximos 30 lotes de fabricação da cor branca, considerando-se os valores medidos pelo espectrofotômetro no qual, seu range de medição varia de 0,0 (zero) a 1,0 (um) quando os produtos estão dentro do especificado, ou seja, estão aprovados. Quando os produtos apresentam medição com valores superiores a 1,0 estão fora do especificado e, consequentemente, reprovados. Essa produção ocorreu no período de 36 dias, na empresa estudada, e os dados foram coletados no laboratório de controle de qualidade. As amostras vindas da produção foram analisadas e comparadas no espectrofotômetro com as cores originais; e os resultados, arquivados por meio de relatórios. Na tabela 1, estão demonstrados os valores coletados no processo de fabricação de laminados sintéticos de cor branca.

Tabela 1. Amostras coletadas na fabricação de laminados sintéticos brancos.

\begin{tabular}{|c|c|c|c|c|c|}
\hline \multicolumn{1}{|c|}{ Amostra } & Valor & \multicolumn{1}{c|}{ Amostra } & \multicolumn{1}{c|}{ Valor } & Amostra & Valor \\
\hline $\mathbf{1}$ & 0,32 & $\mathbf{1 1}$ & 0,25 & $\mathbf{2 1}$ & 0,69 \\
\hline $\mathbf{2}$ & 1,04 & $\mathbf{1 2}$ & 0,13 & $\mathbf{2 2}$ & 0,72 \\
\hline $\mathbf{3}$ & 0,45 & $\mathbf{1 3}$ & 0,35 & $\mathbf{2 3}$ & 0,53 \\
\hline $\mathbf{4}$ & 0,46 & $\mathbf{1 4}$ & 0,79 & $\mathbf{2 4}$ & 1,07 \\
\hline $\mathbf{5}$ & 0,5 & $\mathbf{1 5}$ & 0,65 & $\mathbf{2 5}$ & 1 \\
\hline $\mathbf{6}$ & 0,12 & $\mathbf{1 6}$ & 1,02 & $\mathbf{2 6}$ & 1,06 \\
\hline $\mathbf{7}$ & 0,43 & $\mathbf{1 7}$ & 0,03 & $\mathbf{2 7}$ & 0,41 \\
\hline $\mathbf{8}$ & 0,48 & $\mathbf{1 8}$ & 0,15 & $\mathbf{2 8}$ & 0,08 \\
\hline $\mathbf{9}$ & 0,99 & $\mathbf{1 9}$ & 0,88 & $\mathbf{2 9}$ & 0,46 \\
\hline $\mathbf{1 0}$ & $\mathbf{1}$ & $\mathbf{2 0}$ & 0,97 & $\mathbf{3 0}$ & 0,45 \\
\hline
\end{tabular}

Analisando os dados coletados no processo, quatro amostras ficaram acima do limite superior de especificação, ou seja, 13,3\% da amostra está fora do especificado, demonstrando que o processo tem problemas, instabilidade e potenciais problemas de causa especiais estão afetando este processo.

A segunda etapa do estudo foi a elaboração do histograma. Na elaboração do histograma, Montgomery (2004) sugere que, para amostras pequenas (até 100 amostras), aplique-se a raiz quadrada da amostra, para definir a quantidade de classes do histograma.

Aplicando esse modelo, a amostra tamanho de 30 deve ter 5,4, ou seja, arredondando para cima o histograma deve ter seis classes. Na tabela 2, é apresentado a definição das classes do histograma e a quantidade de amostras que foi classificada em cada intervalo. 
Tabela 2. Frequências dos valores medidos na amostra

\begin{tabular}{|cccccc|}
\hline $\mathbf{N}$ & \multicolumn{2}{c}{ Classes } & Frequência & $\begin{array}{c}\text { Ponto } \\
\text { Médio }\end{array}$ & $\%$ \\
\hline $\mathbf{1}$ & 0,03 & 0,2 & 5 & 0,12 & 17 \\
$\mathbf{2}$ & 0,2 & 0,38 & 3 & 0,29 & 10 \\
$\mathbf{3}$ & 0,38 & 0,55 & 9 & 0,46 & 30 \\
$\mathbf{4}$ & 0,55 & 0,72 & 3 & 0,64 & 10 \\
$\mathbf{5}$ & 0,72 & 0,9 & 2 & 0,81 & 7 \\
$\mathbf{6}$ & 0,9 & 1,07 & 8 & 0,98 & 27 \\
\hline
\end{tabular}

Analisando os dados da frequência das amostras, nota-se que nenhum dos lotes fabricados teve o valor idêntico ao padrão de engenharia, uma vez que o menor valor medido foi 0,03 e o padrão de engenharia é 0,00 . Além disso, percebe-se que a maior frequência de valores ocorreu no intervalo 0,38 a 0,55 e a segunda maior frequência foi nos intervalos de 0,90 a 1,07. Analisando esse comportamento, pode-se perceber que os lotes produzidos têm tendência a se afastar na direção do limite superior de especificação, demonstrando que esse processo pode estar descentralizado.

A próxima etapa consiste na elaboração do histograma. Como esse processo tem uma característica especial de ter somente limite central de especificação e limite central de especificação, o ideal é que o formato do histograma tenha a forma de meia curva normal, o lado positivo da curva de Gauss. No Figura 4 está a representação do histograma.

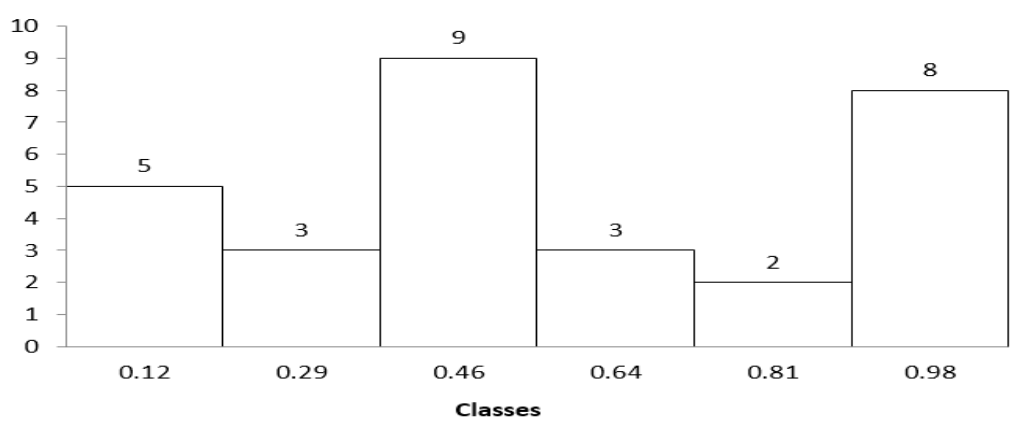

Figure 4. Histograma om os dados coletados no processo de fabricação de laminados sintéticos branco.

Analisando o histograma e considerando que nesse processo o limite central (LC) é o valor 0,0 (zero) e o limite superior de especificação (LSE) é 1,0 (um), fica evidente que sua distribuição não possui uma forma similar do lado positivo da curva de GAUSS, demonstrando que o processo deve ter instabilidade e não deve ter capacidade e/ou capabilidade $\geq 1,0$.

A próxima etapa realizada foi a elaboração dos gráficos de controle do processo, utilizando-se o gráfico de médias individuais. Para a elaboração dos gráficos, utilizaram-se os parâmetros limite superior de controle, limite superior de especificação, limite central, média e desvio padrão. Abaixo, demonstram-se os valores e as fórmulas utilizadas na construção do gráfico de controle. 


$$
\begin{aligned}
& L C=0,0 ; L S E=1,0 ; \mu=0,58 ; \sigma=0,32 ; L S C=\mu+3 \sigma \\
& L S C=0,58+3 * 0,338=1,58
\end{aligned}
$$

Aplicando-se os valores coletados e calculados, elaborou-se o Figura 5, em que se plotaram os dados coletados no processo.

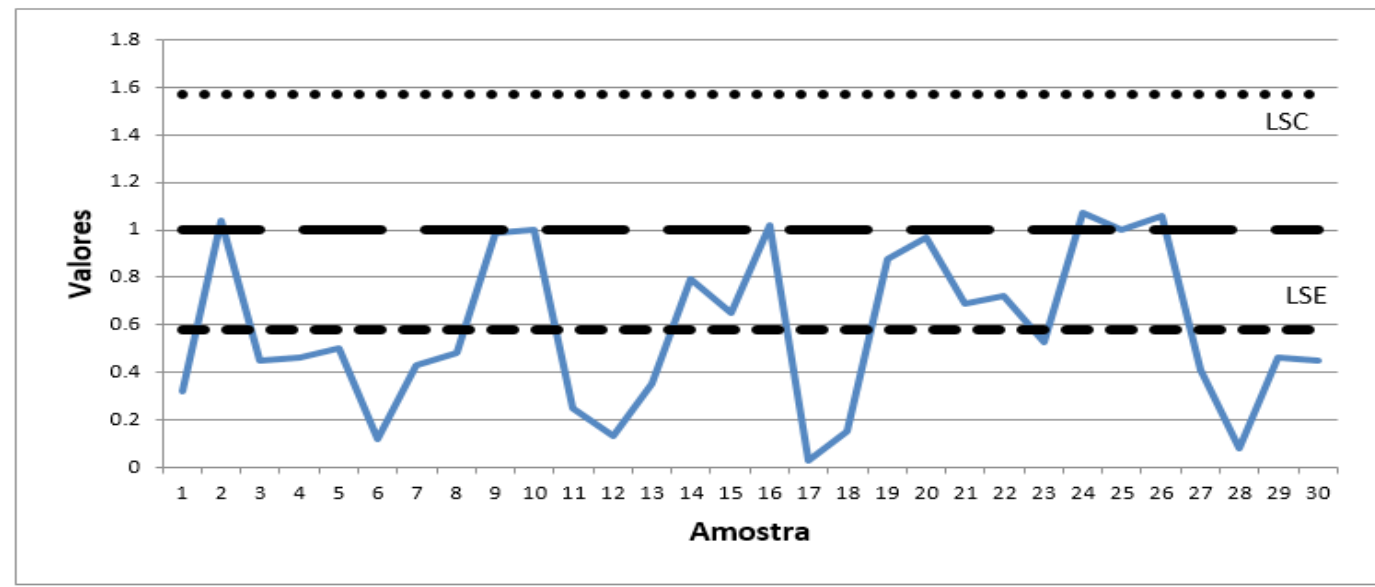

Figura 5. Carta de controle de valores individuais do processo de fabricação

Analisando o gráfico de valores individuais, constata-se que nenhum dos valores está fora dos limites de controle; ou seja, pode-se afirmar que o processo está sob controle estatístico. Contudo, constatou-se que o limite superior de controle é $58 \%$ maior que o limite superior de especificação, demonstrando que os dados coletados têm alta variabilidade.

O próximo gráfico elaborado foi a carta de controle de amplitudes entre as coletas realizadas. Para elaborar este gráfico aplicaram-se as fórmulas descritas a seguir:

$\mathrm{LSC}=\mathrm{R}+3 \sigma ; \mathrm{LIC}=\mathrm{R}-3 \sigma$

$\mathrm{R}=0,315 ; \sigma=0,274$;

$\mathrm{LSC}=0,315+3^{*} 0,274=1,136$;

$\mathrm{LIC}=0,315-3^{*} 0,274=-0,506$, neste caso, utilizar 0,0

Após a análise dos cálculos dos limites de controle para a análise da variabilidade do processo com a utilização do gráfico de amplitude, percebe-se que que o limite de controle inferior do gráfico de amplitude gerou um valor menor que zero, sendo que, nestas situações, deve-se considerar zero como o valor do limite inferior de controle. A figura 6 apresenta a carta de controle de amplitudes. 


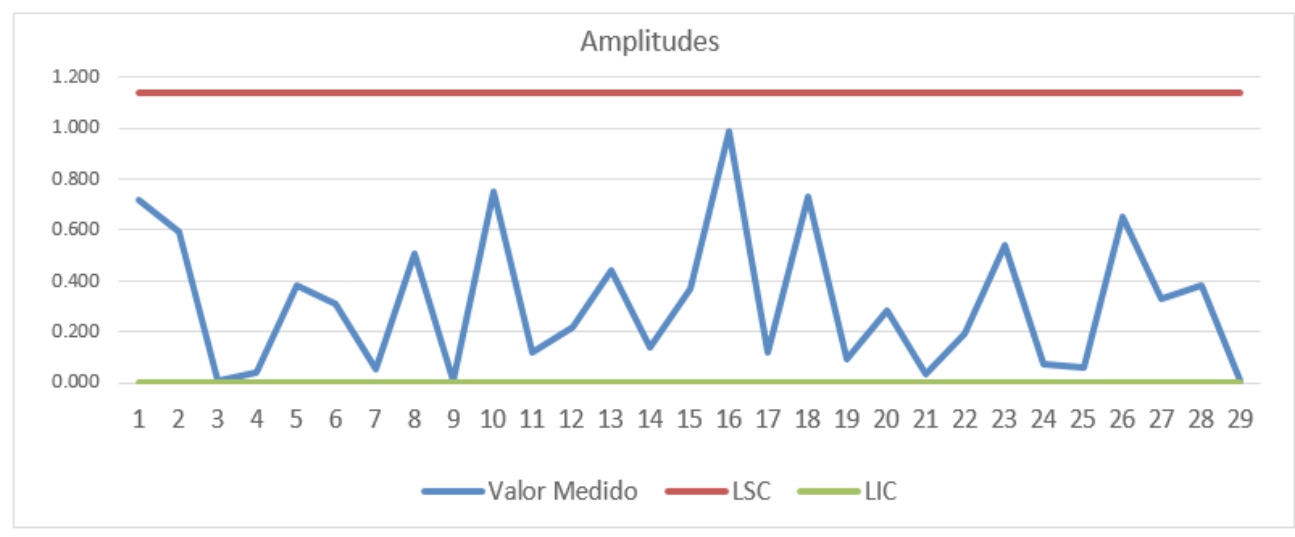

Figura 6. Carta de controle de valores individuais da amplitude processo de fabricação.

Analisando o a carta de controle de valores individuais de amplitude, constata-se que nenhum dos valores está fora dos limites de controle. Ou seja, pode-se afirmar que o processo está sob controle estatístico. Contudo, constatou-se que a amplitude entre a amostra 15 e 16, por exemplo, ocupa todo o limite de especificação dos laminados sintéticos, demonstrando que existe uma variação grande entre os lotes fabricados.

A próxima análise realizada foi a capacidade e capabilidade do processo. Quando estes índices tem resultado $<1,0$, gera um grande índice de material produzido fora do especificado.

Para realizar a análise da capacidade do processo, foi necessário realizar uma adaptação na fórmula tradicionalmente aplicada. Normalmente se aplica, para calcular a capacidade no processo a fórmula onde $\mathrm{Cp}=(($ LSE-LIE) $) / 3 \sigma$.

Essa função deve ser utilizada quando se tem o ajuste na curva de Gauss completa, ou seja, têm-se os limites superior e inferior especificados e os valores coletados superiores e inferiores ao limite central de especificação. Como o processo estudado utiliza o limite central como limite inferior de especificação e se têm dados coletados na parte direita da curva de Gauss, se fez necessária a adaptação da fórmula para cálculo da capacidade do processo, conforme descrita a seguir:

$$
C p=\frac{(L S E-L C)}{3 \sigma}
$$

Considerando a fórmula adaptada do $\mathrm{Cp}$, efetuou-se o cálculo:

$$
C p=\frac{(1-0)}{3 \cdot 0,328}=1,02
$$

A capacidade calculada para esse processo é 1,02, sendo que, valores de $C p>1,0$ significa que o processo tem capacidade. Assim, por esse método, o processo não apresenta problemas, e resultará em índices de rejeição pequenos e não se faz necessário efetuar ação de melhoria sobre ele.

Após a análise do Cp, analisou-se o Cpk do processo com o objetivo de entender se o 
processo que demonstra capacidade está centrado em relação aos limites de especificação. Para realizar a análise do Cpk do processo aplicou-se a fórmula demonstrada abaixo (Montgomery, 2009):

$$
C p k=\frac{(L E S-\mu)}{3 \sigma}
$$

Considerando a fórmula do Cpk, efetuou-se o cálculo.

$$
C p k=\frac{(1-0,583)}{3 \cdot 0,328}=0,424
$$

O Cpk calculado do processo foi < 1,0, ou seja, representa que o processo está descentralizado, gerando perdas por fabricação de materiais fora do especificado, sendo preciso buscar a fonte de variabilidade do processo e agir para aumentar o Cpk, estabilizar o processo e reduzir as perdas.

Como próxima atividade de análise, buscou-se uma ferramenta para entender a causa raiz da variabilidade do processo e, a partir de análises, sugestões e testes de processos, revisar os procedimentos atuais. Também foi verificado se após as melhorias implantadas, os novos resultados demonstram a redução de variabilidade e tornam a capabilidade do processo > 1,0 .

A ferramenta que se utilizou na análise da causa raiz foi o diagrama de causa-efeito, também conhecido como 6M's ou diagrama de Ishikawa, em que se analisam seis fatores que podem ser a fonte da variabilidade do processo. Esses seis fatores são:

1. Mão de obra

2. Método

3. Máquina

4. Material

5. Medida

6. Meio ambiente

Para a execução da análise de causa e efeito, definiu-se que se iriam fabricar dez lotes de laminado sintético de cor branca e monitorar os fatores que poderiam gerar variação, isso com o apoio de uma equipe multifuncional composta por colaboradores da engenharia de produto, da engenharia de processo, da qualidade, da produção, do laboratório e da manutenção.

Quando analisado o fator mão de obra, percebeu-se que os operadores que executaram a operação sempre foram os mesmos nos dez lotes fabricados. Eles executaram a atividade conforme instrução de trabalho, tanto de operação quanto de movimentação da matériaprima e armazenagem do material fabricado.

Quando analisado o fator método, percebeu-se que os documentos que existiam na fabrica, com detalhes do método de trabalho dos operadores, estavam adequados para aquela operação, não sendo os documentos e a execução do método de trabalho a fonte de 
variabilidade.

Analisando o fator máquina, percebeu-se que elas estavam em boas condições de manutenção e utilização, sem alarmes e sem variação de fatores críticos do processo (temperatura, pressão, etc.), sendo descartado esse fator como fonte de variabilidade.

Quando analisado o fator material, visualmente não se percebeu diferença nos lotes de matéria-prima, mas, analisando os certificados enviados pelo fornecedor, estava evidente que os pigmentos brancos enviados eram diferentes lote a lote, sendo que alguns estavam fora do especificado pela engenharia de produto. Esses certificados não eram analisados pelo departamento de qualidade, somente arquivados para registro, sendo que não existia qualquer outro tipo de teste para verificar a especificação de coloração do pigmento.

Quando analisado o fator medida, separaram-se dois lotes de matéria-prima que os certificados afirmavam que estavam dentro do especificado e, após a fabricação com esses lotes, não houve rejeição, eliminando a hipótese de falha no sistema de medição. Ainda tratando das medidas, treinaram-se os inspetores de qualidade para analisar se os materiais recebidos estavam dentro da especificação de processo.

Por último, analisou-se o fator meio ambiente, e nele percebeu-se que, em todos os lotes de produção, o ambiente externo não afetava as condições de operação do equipamento, na máquina e operadores. Por isso esse fator foi descartado como fonte de variação.

Após a análise efetuada, encontraram-se como a única fonte potencial de variabilidade percebida no diagrama de causa e efeito os lotes de matéria-prima fora do especificado. Com isso, definiu-se que se produziriam novos lotes de produção, a partir da segregação de matéria, aplicando somente materiais dentro da especificação de engenharia. Nos próximos 30 lotes de produção, realizou-se o controle de especificação de cor do produto fabricado e obtiveram-se os dados da tabela 3.

Tabela 3. Dados coletados no processo de fabricação após controle dos lotes.

\begin{tabular}{|c|c|c|c|c|c|}
\hline AMOSTRA & VALOR & AMOSTRA & VALOR & AMOSTRA & VALOR \\
\hline $\mathbf{1}$ & 0,56 & $\mathbf{1 1}$ & 0,4 & $\mathbf{2 1}$ & 0,43 \\
\hline $\mathbf{2}$ & 0,6 & $\mathbf{1 2}$ & 0,1 & $\mathbf{2 2}$ & 0,33 \\
\hline $\mathbf{3}$ & 0,21 & $\mathbf{1 3}$ & 0,2 & $\mathbf{2 3}$ & 0,1 \\
\hline $\mathbf{4}$ & 0,27 & $\mathbf{1 4}$ & 0,42 & $\mathbf{2 4}$ & 0,58 \\
\hline $\mathbf{5}$ & 0,15 & $\mathbf{1 5}$ & 0,2 & $\mathbf{2 5}$ & 0,43 \\
\hline $\mathbf{6}$ & 0,55 & $\mathbf{1 6}$ & 0,15 & $\mathbf{2 6}$ & 0,34 \\
\hline $\mathbf{7}$ & 0,25 & $\mathbf{1 7}$ & 0,12 & $\mathbf{2 7}$ & 0,11 \\
\hline $\mathbf{8}$ & 0,42 & $\mathbf{1 8}$ & 0,17 & $\mathbf{2 8}$ & 0,19 \\
\hline $\mathbf{9}$ & 0,28 & $\mathbf{1 9}$ & 0,23 & $\mathbf{2 9}$ & 0,26 \\
\hline $\mathbf{1 0}$ & 0,45 & $\mathbf{2 0}$ & 0,24 & $\mathbf{3 0}$ & 0,49 \\
\hline
\end{tabular}

Como resultado, o processo demonstrou-se mais estável, o que fica evidente nos dados coletados. Por exemplo, a média, antes do controle do lote de matéria-prima era de 0,583 e, no lote de produção controlado, a média demonstrada foi de 0,307 , aproximando a média do limite central de especificação. $\mathrm{Na}$ análise do desvio padrão, ocorreu fato bastante similar. Nos primeiros lotes medidos, o desvio padrão demonstrado foi de 0,328 no lote de produção 
controlado, o desvio padrão reduziu para 0,152 diminuindo mais que $50 \%$ o desvio padrão. Ou seja, fica demonstrada a redução da variabilidade. Outra análise realizada foi a do gráfico da carta de controle de média dos valores individuais. Na figura 7 é apresentado os dados coletados no processo.

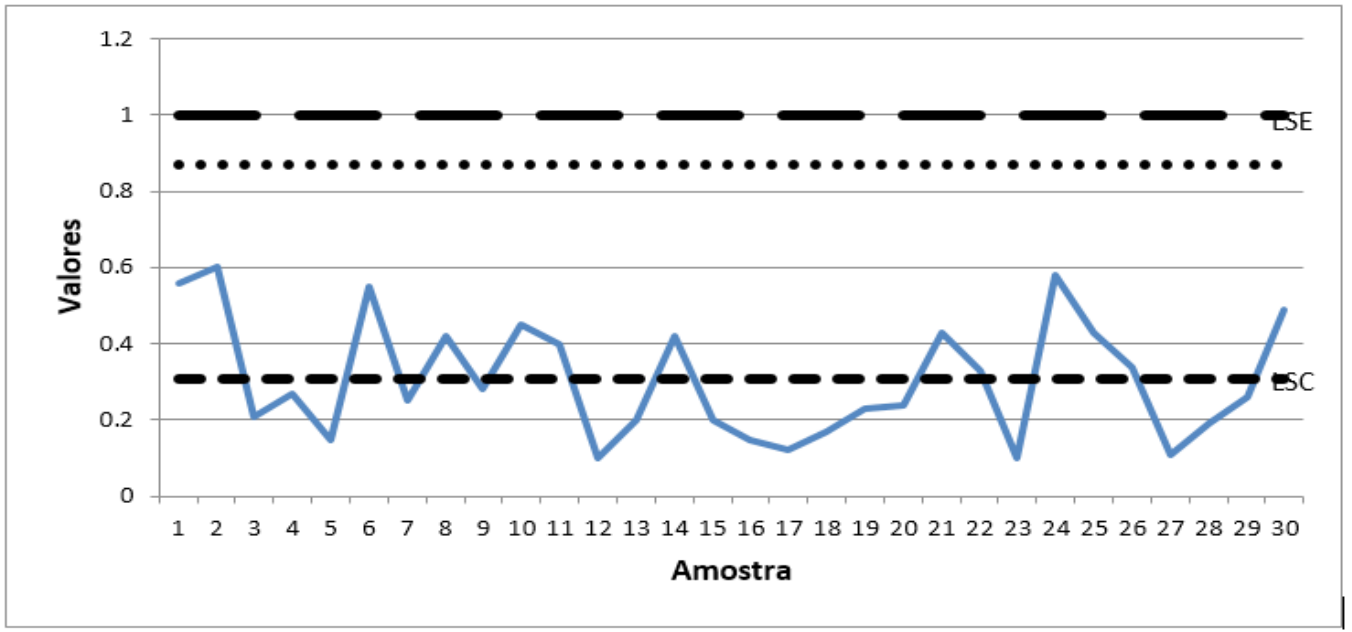

Figura 7. Carta de controle de valores individuais após controle de matéria-prima.

Analisando a figura 7 acima em que a produção foi elaborada com lotes de matéria-prima controlada, e o comparando com os resultados da figura 5, evidencia-se a redução de variabilidade dos valores medidos, a redução da média e nenhum valor medido fora dos limites de especificação e de controle, demonstrando um processo mais estável.

Para finalizar a análise do processo com o lote de material controlado, baseando-se nas mesmas fórmulas demonstradas anteriormente, realizaram-se os cálculos do Cp e do Cpk para os novos valores coletados no processo. Os valores obtidos foram:

$$
C p=\frac{(1-0)}{3 \cdot 0,152}=2,19 \quad C p k=\frac{(1-0,307)}{3 \cdot 0,152}=1,52
$$

Analisando os resultados demonstrados constata-se a melhora significativa da capacidade e da capabilidade do processo. Analisando-se individualmente o $\mathrm{Cp}$, pode-se afirmar que esse processo tem capabilidade $6 \sigma$, porque o índice calculado $(2,19)$ possui é $\mathrm{Cp}>2,0$. Averiguando-se o Cpk, nota-se teve seu índice triplicado, se comparado com os dados originais do processo. Como o índice ficou acima de 1,0 fica demonstrado que o processo tem capabilidade após a utilização da matéria-prima controlada.

Contudo, como o índice Cpk é menor que o índice $\mathrm{Cp}$, o processo se mostra ainda descentralizado. Após as análises, pode-se afirmar que a maior fonte de variação do processo foi descoberta e eliminada, porque todos os indicadores relacionados ao controle estatístico de processo tiveram melhoras significativas. 


\section{DISCUSSÃO DOS RESULTADOS}

Na opinião de Goulart et al. (2010), a qualidade deixou de ser um diferencial e tornou-se um dos pré-requisitos obrigatórios nos diversos setores da economia, e sua busca envolve todos os processos organizacionais, exigindo o comprometimento total dos diversos níveis hierárquicos da organização.

Em alguns processos de fabricação, quando um produto defeituoso é gerado, todos os produtos produzidos a seguir são defeituosos, até que o problema seja resolvido. Foi o que aconteceu neste estudo. Foi identificado um problema, cores brancas com alto índice de rejeição e com valores de amostragem fora dos limites de especificação. Após essa verificação, uma equipe foi em busca de um possível problema, e, sendo aplicadas ferramentas padronizadas de análise de problemas, identificou-se que se tratava de lotes de pigmento branco fora da especificação de qualidade.

Com essa análise, foi possível observar que o processo da empresa estava com sério problema de matéria-prima. Em diversos momentos, os limites ultrapassaram os limites superiores de especificação e se mantiveram afastados do limite central de controle. Ele foi de extrema importância para a empresa, que teve como identificar a causa de um problema e eliminá-lo, gerando redução de perdas. A análise dos dados proporcionou conhecimentos e uma melhoria no processo. Esses achados corroboram a proposição de Paladini (2000) que afirma que, a partir da geração de novos conhecimentos, é possível se obter melhoria da qualidade.

Participaram do estudo alguns funcionários do controle de qualidade, engenharia e produção, laboratório e manutenção. A participação dos colaboradores de vários departamentos gerou uma sinergia entre áreas e conhecimentos dissipados entre departamentos. A experiência e o conhecimento dos envolvidos na análise apoiaram a solução dos problemas e geraram a oportunidade de eles desenvolverem conhecimento sobre um método padronizado para análise de processos.

Ficou evidente, no processo, que a aplicação de métodos padronizados e altamente validados traz resultados para a organização. Nesse caso, a aplicação das ferramentas do CEP gerou a oportunidade de estruturar os dados, interpretá-los e gerar análises, possibilitando a análise das etapas do processo e a percepção do problema que afetava o processo produtivo de fabricação de laminados sintéticos.

A opção aplicada nesta pesquisa de ferramenta para a análise de causa raiz foi o $6 \mathrm{M}$, ou também conhecido como diagrama de causa e efeito. A aplicação dele é bastante difundida e muito bem aceita, mas esta ferramenta tem as suas limitações por ter um desenvolvimento qualitativo. A análise qualitativa baseia-se muitas vezes nas experiências dos envolvidos, sendo que algumas vezes pode limitar a análise e não ser considerando situações que podem ser relevantes para a solução do problema.

Os resultados encontrados pela presente pesquisa corroboram os achados de pesquisas prévias realizadas em outros contextos. Para Gejdos (2015) e Bracke \& Backes (2015) as técnicas de CEP, os índices de capabilidade e a ferramenta de histograma devem ser usados para identificar falhas e estabilizar o processo. A presente pesquisa também identificou que a combinação dessas técnicas supracitadas, possui potencial de reduzir a variabilidade de processos impactando internamente e externamente à empresa. 
Uma das limitações da análise da estabilidade do processo pelas cartas de controle são a limitação da interpretação das causas da variabilidade do processo. Mesmo o processo estando sob controle estatístico, não pode afirma-se que somente causas comuns estão agindo sob aquele processo, mas pode ter alguma causa especial de pouca influência, que se agravada, pode gerar problemas para a organização.

Analisando os comentários de Müller et al. (2015), percebe-se que se obteve a melhoria dois resultados do processo da qualidade operacional, simular a outros casos estudados. As aplicabilidades das análises e das ferramentas do CEP contribuem de forma quantitativa para a melhoria dos resultados e aspectos do processo.

Outro ponto relevante é que, muitas vezes, as pessoas envolvidas nas etapas do processo não têm consciência da importância da sua atividade para a organização nem conhecimento das atividades que precisam desenvolver no seu processo. Mesmo sabendo como a atividade precisa ser desenvolvida e como suas rotinas estão descritas de maneira adequada, muitas vezes, os funcionários não o fazem, devido a não entenderem a importância da atividade e pela falta de monitoramento da gestão sobre a execução dos processos e as atividades desenvolvidas por essas pessoas.

Para se evoluir nesse sentido, os modelos de gestão precisam cada vez mais realizar o monitoramento dos processos e das pessoas, ou seja, o controle das atividades necessita ser realizado in loco, e esse procedimento pode ser revisto após o colaborador entender a importância da sua atividade e compreender que as atividades realizadas no processo são fundamentais para o bom andamento de toda a organização. Com isso, finaliza-se a análise dos resultados coletados, salientando a importância de se utilizarem métodos padronizados para a realização de análises de processos e citando a importância da gestão nos processos organizacionais.

\section{CONSIDERAÇÕES FINAIS}

As conclusões obtidas, a partir deste estudo de caso, evidenciam a importância de se utilizarem métodos padronizados para tratar problemas, mesmo que seja aparente a causa que geradora do problema, reduzindo o risco de tomar decisões baseadas em experiências empíricas.

Essa pesquisa fornece diversas contribuições práticas e acadêmicas não só para a área de conhecimento que envolve as indústrias calçadistas, como também para os processos de identificação de cores por espectrofotômetro e para o campo de investigação do Controle Estatístico de Processos. Em primeiro lugar, esse estudo estende o atual quadro de conhecimento em específico sobre a redução de problemas de qualidade nos processos produtivos de laminados sintéticos por variação de coloração da matéria prima. Portanto, outras empresas desse setor podem se beneficiar dos resultados obtidos na presente pesquisa, ao seguir as etapas do método de trabalho que foram adotados nesse artigo.

Outra contribuição da pesquisa diz respeito ao delineamento metodológico do estudo que foca em particular para um tipo de empresas e de processos. Nesse caso as empresas calçadistas que possuem processo de avaliação de cores de laminados sintéticos. A falta de pesquisa nesse assunto foi corroborada pelas buscas nas bases de dados cientificas. Assim, esse estudo pode orientar investigadores a melhor compreender esse fenômeno resultado 
em melhoria da competitividade da indústria calçadista que sofre com o impacto do mercado asiático.

As implicações da pesquisa permitiram apontar que a aplicação das ferramentas do CEP no processo de qualidade de cores demonstrou ser eficaz para reduz o índice de rejeição, através de uma metodologia estruturada. Tal metodologia trouxe a possibilidade de perceber a fonte do problema que gerava a principal causa de variabilidade do processo. Entre os resultados obtidos da pesquisa foi possível estabelecer a redução dos índices de rejeição do processo para zero defeito, e a melhoria no índice do capabilidade de 0,424 para 1,52. Assim sendo, tais significativos resultados demonstraram redução das fontes de variação do processo produtivo e atingindo-se os objetivos da pesquisa.

As implicações da pesquisa permitiram ainda evidenciar ainda que o incremento de capacidade do processo (CP) e de capabilidade de processo (CPk) no processo foram obtidos através da análise adequada dos processos e utilização das ferramentas da qualidade. Da mesma maneira, também se percebeu que as empresas desse segmento devem explorar o uso dos recursos internos disponíveis, e do conhecimento dos seus colaboradores, visando a manterem-se competitivas no mercado atual e a alcançar sua perpetuação. As ferramentas de qualidade podem ser uma boa estratégia nessa direção. Essa conclusão é corroborada na medida em que a carta de controle estatístico de valores individual utilizada possibilitou que fosse identificado um grave problema no processo de liberação de cor.

Assim sendo, em uma perspectiva geral, é possível dizer que os principais resultados da pesquisa foram o ganho de conhecimento, a melhoria do processo e o aumento da qualidade do produto. Portanto, as ações implantadas neste processo foram expandidas para todos os materiais comprados pela organização, gerando melhoria significativa no processo. Como resultado principal, obteve-se uma redução dos lotes de produção fora do especificado (obtendo zero lotes reprovados), reduzindo seus custos de fabricação e a tornando mais competitiva para encarar a concorrência e sobreviver a toda competição que a indústria está inserida. Como trabalhos futuros, fica a possibilidade de tratar as rejeições de todas as cores de laminado sintético, utilizando o mesmo método e ferramentas para buscar a redução das rejeições e consequentemente a redução de custo.

\section{REFERÊNCIAS}

ALENCAR, J.R. B.; SOUZA, J. M. B.; ROLIM NETO, P. J. and LOPES, C. E. Uso de Controle Estatístico de Processo (CEP) para avaliação da estabilidade e validação da fase de compressão de formas farmacêuticas sólidas. Acta Farm. Bonaerense, 2005, 23(3), 426-435.

ASSINTECAL - Associação brasileira de empresas de componentes para couro, calçados e artefatos. [online]. 2017. [cit. 2016-05-10]. Disponível em: /www.assintecal.org.br>.

ABICALÇADOS - Associação brasileira das indústrias de calçados. [online]. 2017. [cit. 201605-15]. Disponível em: /www.abicalcados.com.br/relatorioanual/.

BRACKE, S. and BACKES, B. Multidimensional analysis of manufacturing processes: process capability within the case study shape drill manufacturing. IFAC, 2015, 48 (3), 2380-2386.

FARIAS, J. P. da S. Avaliação da Satisfação do cliente: Uma experiência com a empresa 
Raboni calçados. Universidade estadual da Paraíba, campus VII, Centro de Ciência Exatas e Sociais Aplicadas, Paraíba, 2012.

GEJDOS, P. Continuous Quality Improvement by Statistical Process Control. Procedia Economics and Finance, 2015, 34, 565-572.

GOULART L.E.T and BERNEGOZZI R.P. O uso das ferramentas da qualidade na melhoria de processos produtivos. XVI International Conference on Industrial Engineering and operations Management: ICIEOM, São Carlos, 2010.

MANCUSO, A.C.B and WERNER, Liane. Estudo dos métodos de previsão de demanda aplicado em uma empresa de auditorias médicas. Revista Ingeniería Industrial, 2014, 13 (1), 99-111.

MIGUEL, P. C and FLEURY, A.C. Metodologia de pesquisa em engenharia de produção e gestão de operações. 2. Ed. Rio de Janeiro: Elsevier, 2010. ISBN 978-8535248913.

MONTGOMERY, D.C. Estatística Aplicada e Probabilidade para Engenheiros. 4. Ed. Rio De Janeiro: LTC, 2009. ISBN 978-8521632412.

MONTGOMERY, D.C.; RUNGER, G.C.; HUBELE, N.F. Estatística aplicada à engenharia. 2. ed. Rio de Janeiro: LTC, 2004. ISBN 978-8521613985.

MÜLLER, P.E.; ROSA, A.; KRUMMENAUER, L.G.; PACHECO, D.A.J.; JUNG, C.F and CATEN, C.S. Contribuições do CEP para a melhoria do desempenho do pós-vendas na indústria calçadista. Revista Ingenieria Industrial, 2015, 14 (1), 51-66.

PALADINI, P.E. Gestão da qualidade: Teoria e Prática. 3 Ed. São Paulo: Atlas, 2000. ISBN 978-8522471157.

PORTERO, P.P. Avaliação instrumental da correspondência de cor de resinas compostas em função de escalas de referência, tonalidade, composição e espessura. Dissertação de Mestrado, UNESP - Universidade Estadual Paulista de Araraquara, Araraquara, 2010. 